\title{
Acupuncture for patients with mild to moderate Alzheimer's disease: a randomized controlled trial
}

\author{
Yujie $\mathrm{Jia}^{\dagger},{ }^{\prime}$ Xuezhu Zhang ${ }^{\dagger}$, Jianchun Yü, Jingxian Han, Tao Yu, Jiangwei Shi, Lan Zhao and Kun Nie
}

\begin{abstract}
Background: Alzheimer's disease (AD) is the most common cause of dementia. However, none of medical treatment can stop or reverse the underlying neurodegenerative of $A D$ at present. Acupuncture has attracted more and more attention in recent years due to its efficacy and very few side effects. Lately, a systematic review has thought that the evidence on the effectiveness of acupuncture in improving the cognitive function of AD patients was not powerful enough. Therefore, the aim of this study is to explore the efficacy and safety of acupuncture in patients with mild to moderate AD.
\end{abstract}

Methods: This was a randomized, controlled, parallel-group, exploratory study with 4-week baseline (T0), 12week treatment phase (T1) and 12-week follow-up period (T2). Patients with mild to moderate AD meeting the included criteria were randomly allocated into either acupuncture or donepezil hydrochloride groups. The acupuncture group(AG) was given acupuncture treatment three times per week and the donepezil hydrochloride group(DG) group was administered donepezil hydrochloride once daily $(5 \mathrm{mg} /$ day for the first 4 weeks and $10 \mathrm{mg} /$ day thereafter). Primary efficacy was measured using Alzheimer's disease Assessment Scale-Cognitive (ADAS-cog) and Clinician's Interview-Based Impression of Change-Plus (CIBIC-Plus). The second outcomes were measured with 23-Item Alzheimer's disease Cooperative Study Activities of Daily Living Scales (ADAS-ADL ${ }_{23}$ ) and Neuropsychiatric Index (NPI).

Results: Of 87 participants enrolled in the study, 79 patients finished their treatment and follow-up processes. The ADAS-cog scores for AG group showed obvious decreases at T2 and $\Delta(T 2-T 0)$ when compared with DG group, and significant between-group differences were detected (all $p<0.05$ ). The mean CIBIC-Plus values for the AG group at $\mathrm{T} 1$ and $\mathrm{T} 2$ were much lower than that for the DG group, and there were significant differences between the two groups ( $<0.05)$. There were no significant between-group differences in the scores of ADAS-ADL 23 and NPI during the study period. Treatment discontinuations due to adverse events were $0(0 \%)$ and 4 (9.09\%) for the $A G$ and DG groups, respectively.

Conclusions: Acupuncture is safe, well tolerated and effective in improving the cognitive function, global clinical status of AD.

Trial Registration: ChiCTR-IOR-17010465 (Retroactively registered on 18 JAN 2017).

Keywords: Acupuncture, Alzheimer's disease, Randomized controlled trial

\footnotetext{
* Correspondence: yujianchun1964@126.com

${ }^{\dagger}$ Equal contributors

First Teaching Hospital of Tianjin University of Traditional ChineseMedicine,

Tianjin 300193, China
} 


\section{Background}

Alzheimer's disease (AD) is a slowly progressive neurodegenerative disorder characterized by memory impairment and other cognitive disabilities, personality changes [1].AD is the most common cause of dementia, accounting for $60 \sim 70 \%$ of all dementia cases [2].Age is the most important risk factor for $\mathrm{AD}$. The prevalence of $\mathrm{AD}$ rises exponentially in elderly people from $1 \%$ at age of 65 to approximately $40-50 \%$ by the age of 95 [3].Moreover, the health care costs for $\mathrm{AD}$ are expensive, which have reached $\$ 172$ billion per year in the United States alone [4].It is estimated that $60 \%$ of $\mathrm{AD}$ patients live in developing countries, with the proportion being raised to more than $70 \%$ by 2040 [5].It can be predicted that AD represents a major public health challenge as a consequence of rapid increase in the aging population worldwide, especially in developing countries.

More and more people pay attention to $\mathrm{AD}$ because the decline in memory and other cognitive functions leads to a loss of independence which has a wideranging impact on individuals, families and healthcare systems [6].Thus, there is a need for prospective studies to clarify the pathogenesis of this condition and to provide appropriate measures for prevention and treatment of AD. Although AD had already been described about 100 years ago and we have paid enormous research efforts, at present none of medical treatment can stop or reverse the underlying neurodegenerative of $\mathrm{AD}$ [7].The medications currently approved by the Food and Drug Administration and European Agency for the Evaluation of Medical Products include cholinesterase-inhibitors $(\mathrm{CI})$ and memantine, which offer modest symptomatic relief [8].A systematic review of the literature has revealed that CI resulted in significantly higher rates of adverse events and discontinuation of treatment [9].At the same time the cost effectiveness of the currently available therapeutic agents for $\mathrm{AD}$ has undergone great scrutiny and remains controversial, especially outside the united states of American(USA) [10]. So we should find a treatment that will not only yield maximum benefits for individual patients across multiple domains, including cognition, daily function and behavior, but also provide realistic expectations for patients and caregivers throughout the course of the disease [11].

Acupuncture, as a style of traditional Chinese medicine(TCM), has attract more and more attention in recent years due to its efficacy and very few side effects [12]. However, a new systematic review has thought that the evidence on the efficacy of acupuncture in improving the cognitive function of $\mathrm{AD}$ patients was not powerful enough [13]. Another systematic review has also considered that existing evidence didn't demonstrate the effectiveness of acupuncture for AD [12]. Therefore, the randomized, controlled clinical trial was designed to further determine the effectiveness and safety of acupuncture among patients with mild to moderate AD.

\section{Methods}

Trial design, setting, randomization and blinding

The study was a randomized, drug-controlled, parallelgroup trial to determine the efficacy and safety of acupuncture compared with donepezil in patients with mild to moderate AD from November 2015 to May 2016. All participants came from the older residents with dementia in an epidemiological investigation. The survey was carried out in the community of Simianzhong Street, Heping District, Tianjin City from August to October 2015.The study would last 28 weeks: a 4 -week prospective baseline period, 12-week treatment period, and a 12week follow-up period. The cognitive abilities, activities of daily living and behavioral symptoms of patients would be evaluated before treatment, after 12 weeks, and after 24 weeks using a variety of neuropsychological tests. Overall clinical status was measured at week 16, and 28. After the baseline, all patients were randomly allocated into either AG or DG in a ratio of 1:1 by a computer-generated, randomly location sequence (random list generated with SPSS 13.0) under the help of a professional statistician. The random number table was sealed in a special envelope.

In this study, physicians could not be blinded due to the nature of the intervention. The acupuncturists had to know the group assignment because of manipulation. Data collection was performed by 2 blinded evaluators. Blinding was also maintained for data analysis. Apart from the differences in treatment methods between the two groups, all subjects were treated as equally as possible. During the intervention, acupuncturists and data collection staff would visit patients at different time so that they could not exchange information with each other.

The study was performed according to common guidelines for clinical trials (Declaration of Helsinki, International Conference on Harmonization /WHO Good Clinical Practice standards including certification by an external audit).The trial protocol had been approved by the Research Ethical Committee of First Teaching Hospital of Tianjin University of Traditional Chinese Medicine (TYLL2015[K]003) on July 30, 2015.

\section{Participants}

This study included residents aged 50 to 85 years and main types of surveys were home interview. The screening process consisted of the following three phases: the first phase: personal information of the residents (including age, sex, occupation, weight, marital status, education level, previous medical history, family 
medical history, living habits, subjective emotion and combined disease) were collected and neuropsychological scales(Mini-mental State Examination, MMSE and Activity of Daily Living scale, ADL) were evaluated. The second phase: the residents whose MMSE and ADL scores were lower than standard were further assessed using 17-item Hamilton depression scale(HAMD) and Hanchinski ischemia scale(HIS). The third phase: physician of neurology made a diagnosis based on medical history and nervous system medical examination.

Diagnostic criteria designed to identify patients with probable $\mathrm{AD}$ were developed by the Diagnostic and Statistical Manual of Mental Disorders, Fourth Edition and National Institute of Neurological and Communicative Disorders and Stroke-Alzheimer's Disease and Related Disorders Association criteria [1]. The patients included in this study were required to have a MMSE score between 10 and 23 [14], a HAMD score $<7$, a HIS score $\leq 4$, and have a reliable caregiver who is able to accompany the participant to all study visits. Individuals were excluded if they had evidence of other diseases which could lead to dementia other than AD; significant unstable psychiatric or neurologic disorders; clinically relevant uncontrolled or unstable gastrointestinal, renal, hepatic, endocrine or cardiovascular disease; clinically significant obstructive pulmonary disease; B12 or folic acid deficiency; alcoholism or drug misuse or current systemic illness influencing cognitive assessment; a history of hematologic and oncologic disorders in the past 2 years. Subjects could not participate if they had received any treatments which probably improve cognitive function within the past 1 month and known sensitivity to acupuncture or Donepezil Hydrochloride. Patients were also excluded from the study if they were incapable of giving written informed consent from a legal representative of the participants.

\section{Interventions}

The treatment strategies for acupuncture was initiated by Professor Jingxian Han during long theoretical study and clinical experience according to the Sanjiao theory of TCM. Then, three experienced acupuncture specialists (zhilongzhang, jianchunyu, yong tang) discussed and finalized the treatment strategies in a consensus process. The acupuncture therapy was performed by nine acupuncturists who are registered Chinese medicine practitioners of the Ministry of Health of the People's Republic of China and have more than 6 years (median 7.2 years) of clinical experience. The sterile, disposable needles (Huatuo, Suzhou Medical Instruments Factory, China) were used in this study with a diameter of $0.25 \mathrm{~mm}$ and a length of $40 \mathrm{~mm}$. These acupoints were employed as basic acupuncture formulas, including RN17(danzhong), RN12(zhongwan), RN6(qihai), ST36(zusanli), SJ5(waiguan) and SP10(xuehai). Moreover, the following acupoints could be selected as auxiliary acupoints according to patient's symptoms and tongue manifestation,LR3(taichong),GB39(xuanzhong),ST40(fenglong),B L17(geshu),ST44(neiting),ST25(tianshu) andRN4(guan yu an). Except for RN17, RN12, RN6, RN4, the other acupoints were bilateral. Acupuncture prescriptions were individualized to each patient, and different points were used based on the discretion of the acupuncturist.

During acupuncture, patients would have a needling feeling of numbness, tingling, swelling or muscle weakness, which is known as "de qi" and considered as indicators of effective needling. To evoke needle sensation, the needles were inserted obliquely and upward $15 \mathrm{~mm}$ into RN17, 15 25 mm perpendicularly into RN12, RN6 and ST36, then rotated at small-amplitude and highfrequency with reinforcing method for 30s. The needles were inserted perpendicularly $15 \sim 25 \mathrm{~mm}$ into SJ5, then rotated with normal reinforcement and normal reduction method for $30 \mathrm{~s}$. For SP10, the needle was inserted obliquely $15 \sim 25 \mathrm{~mm}$ into the acupoint, then rotated with big-amplitude and low-frequency reducing method for 30s. The needles would be retained in situ for $30 \mathrm{~min}$. Acupuncture treatment was given three times weekly for 12 weeks. Any additional therapies for AD were not permitted during the entire study period, and only necessary explanations were provided to patients to avoid biased data. All acupuncturists received operation instructions from professor Han, a videotape, and a brochure with detailed information on acupuncture treatment. The patients in the DG group received $5 \mathrm{mg} /$ day of donepezil hydrochloride(Aricept ${ }^{\oplus}$, Weicai (China) Pharmaceutical Co., Ltd) for the first 4 weeks and $10 \mathrm{mg} /$ day thereafter [15].

\section{Outcome measures}

The primary outcome measures were (1) ADAS-cog, which consists of 11 tasks to assess multiple cognitive domain including memory, language, praxis and orientation. ADAS-cog score ranges from 0 to 70 with higher scores indicating greater impairment. (2)CIBIC-Plus, which mainly assesses treatment effect on overall clinical status of $\mathrm{AD}$ patients with scores ranging from 1 (marked improved) to 7(markedly worse) [16].

The second outcome measures were (1) ADAS-ADL 23 , which is a test to assess basic and instrumental activities of daily living. The ADAS-ADL ${ }_{23}$ score ranges from 0 to 78 , and higher scores indicate lower levels of activities of daily living [17]; (3) NPI, which is a condition-specific measure to assess frequency and severity of behavioral symptoms in patients with dementia. It covers 10 neuropsychic symptoms and 2 autonomic nervous symptoms with total scores ranging from 0 to 144 (higher numbers indicating more behavioral disturbance) [18]. 
The safety parameters were assessed during each visit, including spontaneously reported adverse events (AEs) or serious AEs (SAEs), vital signs (temperature, heart rate and blood pressure) and physical examination.

\section{Sample size}

According to the results of previous study, an improvement of ADAS-Cog scores for the acupuncture and control groups were 6.99 (standard deviation: 3.23) and 4.02 (standard deviation: 2.11), respectively [19]. Therefore, the number of sample size was calculated using the following formula:

$$
\mathrm{n}_{1}=\mathrm{n}_{2}=2\left[\frac{\left(t_{\alpha}+t_{\beta}\right) s}{\delta}\right]^{2}(\alpha=0.05, \beta=0.10)
$$

As a result, we estimated that 17 patients were required in each group. A 20\% drop-out rate was assumed, therefore, 21 patients should be enrolled in each arm.

\section{Statistical analysis}

The results were presented as mean \pm SD or frequencies and percentages, according to the type of variables. Demographic data were analyzed using standard statistical tests (e.g., t-test, Fisher's exact test and binomial test) according to two-tailed $p$-values. For the betweengroup comparisons at baseline, either the Chi-Square test or one-way covariance was used. Chi square test was applied for count data, and independent samples ttest was used for measurable variables. Efficacy outcome parameters were analyzed on an intent-to-treat basis. The missing data were not disposed. Repeated measures analysis of variance was used to determine whether significant differences exist across time (except CIBICPlus).Two-independent-samples t-test was performed when the difference met normal distribution. All AEs were coded using a World Health Organization (WHO)based dictionary of preferred terms. The numbers (percentages) of patients with at least one $\mathrm{AE}$, at least one $\mathrm{SAE}$, and at least one AE leading to discontinuation were summarized irrespective of relationship to treatment. All safety-related observations were summarized using descriptive statistics. The $P$ values lower than 0.05 were considered to be statistically significant. All statistical analysis was performed using SPSS software version 13.0(SPSS Inc., Chicago, USA).

\section{Results}

\section{Participants}

The flow of participants through the trial was shown in Fig. 1. Form November 2015 to May 2016, 152 patients were screened for eligibility. Of these, 57 could not be included because they did not meet all eligibility criteria. A total of 87 patients were enrolled and randomly

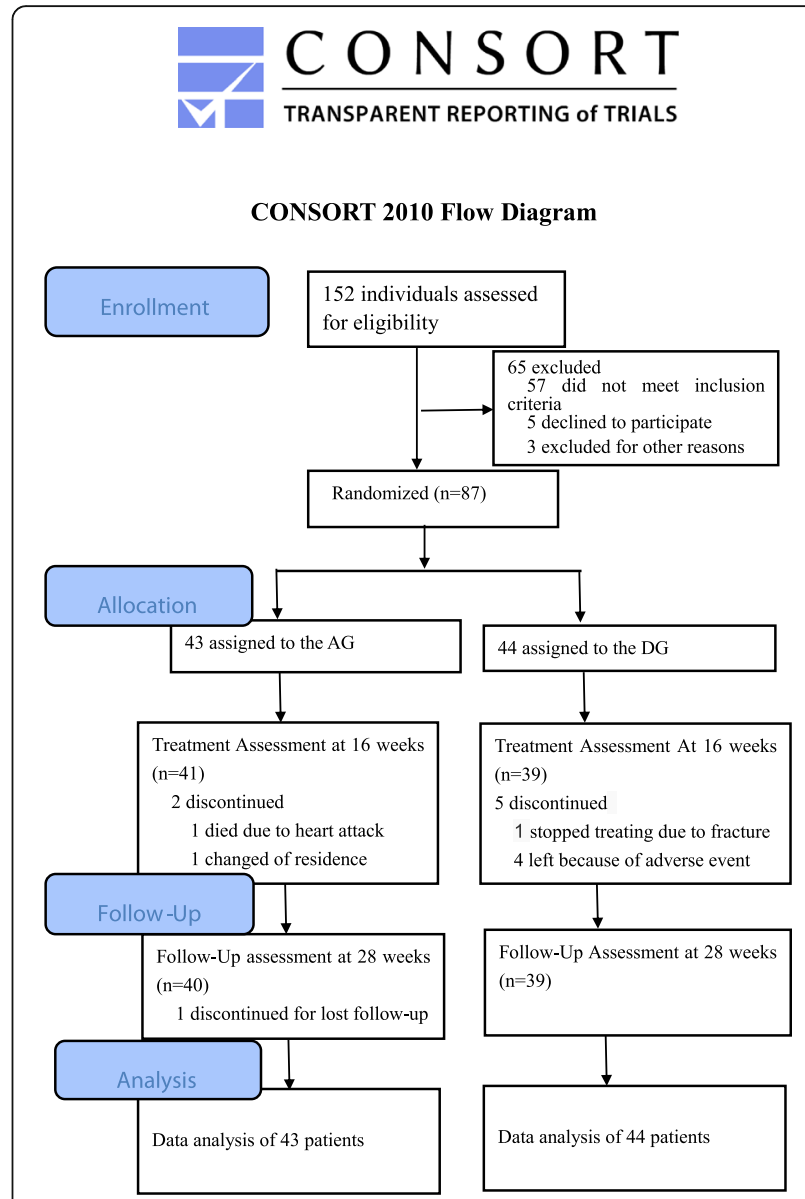

Fig. 1 Flowchart of the study design (based on CONSORT 2010 guidelines)

assigned to either AG $(n=43)$ and DG $(n=44)$ groups. Eight $(9.19 \%)$ out of 87 patients dropped out during the trial, one patient stopped the treatment due to fracture, one patient died from heart attacks, four patients could not tolerate donepezil for SAEs, one patients withdrew owing to the change of address, and one patient lost at the follow-up. A total of 79 patients $(90.80 \%)$ completed the entire treatment and follow-up processes, 40 $(93.02 \%)$ in the AG and 39(88.64\%) in the DG.

There were no significant differences in the demographic characteristics (age, sex, education year, marital status, et al) and baseline efficacy parameters (ADAS$\operatorname{cog}, \mathrm{ADCS}-\mathrm{ADL}_{23}$ and NPI) of the subjects initially enrolled in the study between the two patient cohorts (Table 1).

\section{Outcome measures}

Mean scores of ADAS-cog, CIBIC-Plus, ADCS-ADL 23 and NPI at all time points and the change scores between baseline (T0) and week16 (T1), and between T0 and week 28 (T2) for the two groups were shown in Table 2. 
Table 1 Baseline characteristics of study participants

\begin{tabular}{|c|c|c|c|}
\hline & Acupuncture & Donepezil & total \\
\hline \multicolumn{4}{|l|}{ Gender } \\
\hline Men n (\%) & 13(30) & $16(36)$ & 29(33) \\
\hline women n (\%) & $30(70)$ & $28(64)$ & $58(67)$ \\
\hline Age, y, M (SD) & $75.11(6.53)$ & $74.50(6.83)$ & \\
\hline \multicolumn{4}{|l|}{ Education(year) } \\
\hline On (\%) & $6(14)$ & $6(14)$ & $12(14)$ \\
\hline $1-6$ n (\%) & $6(14)$ & $10(23)$ & 16(18) \\
\hline 7-9 n (\%) & $22(51)$ & $20(45)$ & $42(48)$ \\
\hline$\geq 10$ n (\%) & $9(21)$ & $8(18)$ & $17(20)$ \\
\hline \multicolumn{4}{|l|}{ Severity of dementia } \\
\hline Mild n (\%) & $24(56)$ & $23(52)$ & $47(54)$ \\
\hline Moderate n (\%) & $19(44)$ & $21(48)$ & $40(46)$ \\
\hline $\begin{array}{l}\text { times since symptoms } \\
\text { first started, } M(S D)\end{array}$ & $4.46(0.99)$ & $4.42(1.06)$ & \\
\hline \multicolumn{4}{|l|}{ Associated diseases n (\%) } \\
\hline Hypertension & $19(44)$ & 17(39) & $36(41)$ \\
\hline Diabetes & $15(35)$ & $13(30)$ & $28(32)$ \\
\hline Coronary heart disease & $12(28)$ & $14(32)$ & $26(30)$ \\
\hline Insomnia & $10(23)$ & $13(30)$ & 23(26) \\
\hline Constipation & $12(28)$ & $9(20)$ & $21(24)$ \\
\hline Benign prostatic hyperplasia & $7(16)$ & $9(20)$ & $16(18)$ \\
\hline Osteoarthritis of the knee & $4(9)$ & $5(11)$ & $9(10)$ \\
\hline ADAS-cog score, mean (SD) & $29.38(9.43)$ & $30.15(9.16)$ & \\
\hline ADCS-ADL23 score, mean (SD) & 49.44(11.62) & $50.13(13.57)$ & \\
\hline NPI score, mean (SD) & $9.28(2.49)$ & $8.97(2.69)$ & \\
\hline
\end{tabular}

The results of repeated measures analysis for ADAScog scores showed a significant time effect $(P=0.000)$ and time $\times$ group interaction $(P=0.000)$, but no group effect $(P=0.235)$ were observed. There were significant between-group differences in ADAS-cog scores at T2 and $\Delta$ (T2-T0) (all $p<0.05$ ), but no statistical differences were found at other time points (Fig. 2a).
The mean CIBIC-Plus values were 3.07 (1.03) and 4.08 (0.81) for the AG group at T1 and T2, which were much lower than that for the DG group. The CIBIC-Plus scores showed obvious decreases for AG group when compared with DG group, and significantly statistical differences were observed between the two groups $(P<0.05)$ (Fig. 2b).

There were a time effect $(P=0.000)$ were found for the ADCS- $\mathrm{ADL}_{23}$ scores analyzed using repeated measures approach, but no treatment $\times$ time interaction $(P=0.421)$ and group effect $(P=0.758)$ were detected. No between-group differences were found in ADCS$\mathrm{ADL}_{23}$ scores at any time points, and the change scores didn't have statistical significance between the two groups (Fig. 2c).

There was a time effect $(P=0.000)$, but no treatment $x$ time interaction $(\mathrm{P}=0.05)$ and treatment $\operatorname{effect}(P=0.402)$ were found in the NPI scores determined with the repeated measures approach. There were not any significant between-group differences in NPI scores at any time points, and the change scores also didn't have statistical significance between the two groups(Fig. 2d).

\section{Safety and tolerability outcomes}

During acupuncture treatment, 4 patients $(9.3 \%)$ experienced punctate hemorrhage after the needles were taken out (we stopped the bleeding by pressing for 5-10 s with the help of sterile dry cotton), and 1patient(2.3\%) had bruising. No serious adverse events were reported and no patients withdrew from the trial. During donepezil treatment, 7 patients(15.9\%) complained adverse events, including dizziness, nausea, loss of appetite, diarrhea, constipation, fatigue and agitation. In the 7patients, 4 cases $(9.09 \%)$ withdrew from the trial and the others that had mild reaction continued to receive treatment until the end of follow up.

\section{Unexpected finding}

In the AG group, 5 cases of insomnia, 4 cases of constipation, 6 elderly male cases with benign prostate

Table 2 Mean scores at all time points and change scores for ADAS-Cog, CIBIC+, ADCS-ADL 23 and NPI

\begin{tabular}{lllllll}
\hline & Group & $\begin{array}{l}\text { T0 } \\
\text { baseline }\end{array}$ & $\begin{array}{l}\text { T1 } \\
16 \text { weeks }\end{array}$ & $\begin{array}{l}\text { T2 } \\
28 \text { weeks }\end{array}$ & $\Delta($ T1-T0) & $\Delta($ T2-T0) \\
\hline ADAS-cog & AG & $29.38(9.43)$ & $25.30(11.33)$ & $26.28(10.73)^{a}$ & $4.08(7.73)$ & $3.10(6.01)^{a}$ \\
& DG & $30.15(9.16)$ & $28.18(9.78)$ & $31.25(10.71)$ & $1.97(2.08)$ & $-1.10(3.42)$ \\
CIBIC-Plus & AG & $\mathrm{N}$ & $3.07(1.03)^{a}$ & $4.08(0.81)^{a}$ & $\mathrm{~N}$ & $\mathrm{~N}$ \\
& DG & $\mathrm{N}$ & $3.69(0.95)$ & $4.92(0.77)$ & $\mathrm{N}$ & $\mathrm{N}$ \\
ADCS-ADL23 & AG & $49.44(11.62)$ & $47.85(11.22)$ & $48.18(11.32)$ & $1.59(6.11)$ & $1.26(6.54)$ \\
& DG & $50.13(13.57)$ & $48.20(13.16)$ & $49.43(13.45)$ & $1.92(3.03)$ & $0.69(3.05)$ \\
NPI & AG & $9.28(2.49)$ & $7.25(2.69)$ & $8.13(2.78)$ & $2.02(0.59)$ & $1.15(0.60)$ \\
& DG & $8.97(2.69)$ & $7.61(2.30)$ & $9.31(2.42)$ & $1.36(0.57)$ & $-0.33(0.58)$ \\
\hline
\end{tabular}

${ }^{a}$ indicates significance between the two study groups $(p<0.05)$

$\mathrm{N}$ indicates not determined 
A

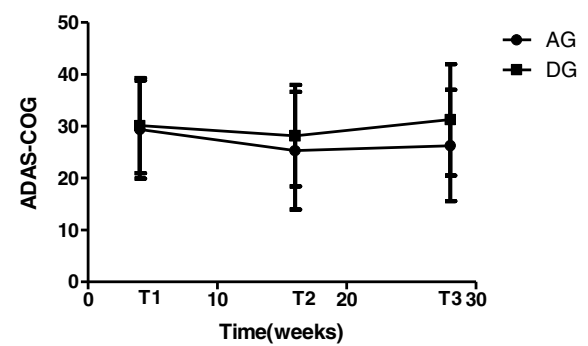

C

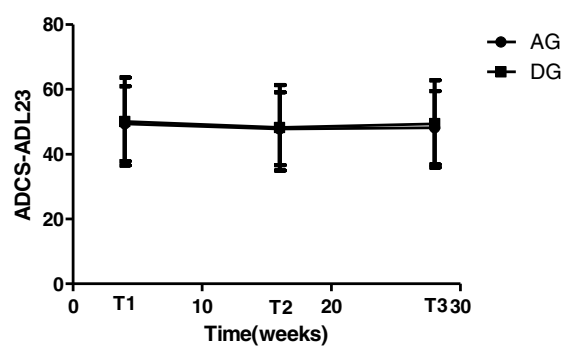

B

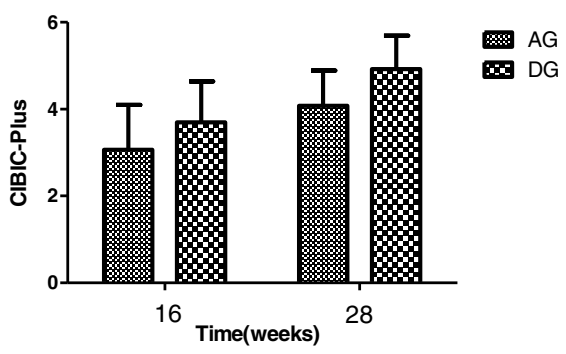

D

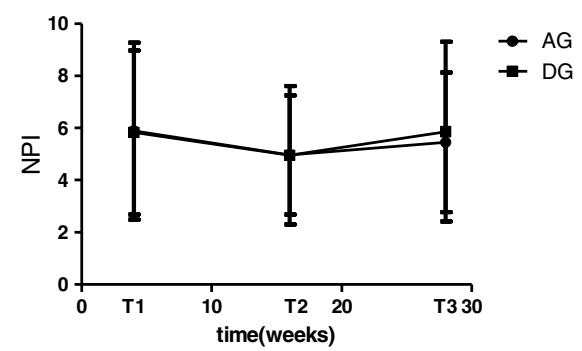

Fig. 2 Scores of ADAS-cog, CIBIC-plus, ADCS-ADL23, NPI in AG and DG

hyperplasia and 2 cases of knee arthritis reported that their symptoms were obviously improved. However, patients in the DG group did not demonstrate equivalent improvements.

\section{Discussion}

This exploratory, 28-week, controlled study was designed to examine the efficacy of acupuncture in the patients with mild-to-moderate AD compared to donepezil. The results indicated that acupuncture could improve cognitive function, global clinical status according to the scores of ADAS-cog, CIBIC-Plus. But the effect of acupuncture to improve activities of daily living and behavioral symptoms seemed to be limited based on the scores of ADCS$\mathrm{ADL}_{23}$ and NPI.

In spite of long history and public acceptance of acupuncture, an unequivocal scientific explanation regarding to the physiological mechanism of acupuncture has not been obtained and awaits further investigation. In recent years, many research works have been done in order to offer probable evidence and promote the therapeutic use of acupuncture. For example, some studies have proven that acupuncture could enhance functional connectivity between the hippocampal regions [20],activate certain cognitive-related regions [21] and modulate default mode network activity [22] in AD patients using neuroimaging techniques. After stimulating HT7 by laseracupuncture, acetylcholinesterase activity was significantly suppressed in the hippocampus of memoryimpaired rats [23]. Electro-acupuncture at DU20 could markedly ameliorate cognitive impairments, reduce aberrant overexpression of $\beta$-amyloid ${ }_{1-42}$, and inhibit neuronal apoptosis, significantly enhance expression levels of mature brain-derived neurotrophic factor (BDNF) and pro-BDNF in APP/PS1 mice [24]. In addition, some experiments have shown that acupuncture could improve the learning and memory abilities of senescence-accelerated mouse 8 by inducing cell proliferation in different brain regions [25], up-regulating triose phosphate isomerase activity in hippocampal tissues [26], modulating synapse functions relevant to cytoskeleton and inducing neurotransmitters secretion [27].

Furthermore, nonspecific physiological effects of acupuncture had also been reported, including neurophysiological and neuro-chemical responses $[28,29]$. In this study, we did not use sham acupuncture to exclude possible placebo effects, because it is not usually appropriate to use sham acupuncture in a pragmatic trial due to a likely detrimental effect on the trial's ecological validity [30]. For this reason, donepezil was used as control medicine and a direct head-to-head comparison between acupuncture and donepezil was established in this study to confirm the efficacy of acupuncture in AD patients. In china, some similar studies have been done and the results have proven that acupuncture treatment was similarly effective as medication and side effects of acupuncture were far fewer than medicine. However, the results of these studies were not fully consistent and the design of the clinical trials is not very rigorous. The results of this study were substantially consistent with 
the published literatures [19].In this study, only minor adverse effects were observed and no patients withdrew due to side effects. However, our results could not provide evidence to prove that acupuncture therapy is better in safety than donepezil due to the small sample size. High dropout rate due to adverse effects have been reported in clinical trials with donepezil. For example, the dropout rate was $9.3 \%$ in a 15 -week, double-blind, placebo-controlled study on donepezil [31]. In another open-label, multicenter, randomized trial of donepezil, the withdraw rate owing to the same reason was as high as $17 \%$ [32].In spite of minor adverse effects and low dropout rate, acupuncture could also provide some benefits for other diseases based on the theory of syndrome differentiation and treatment of TCM.

We must also note several potential limitations in this study. Firstly, the small sample size leads to underpowered results. Secondly, participants who glad to take part in this study seemed to have a positive attitude toward treatment (including acupuncture and medicine). It was possible that patients with high treatment expectations will introduce positive bias into the results of the study. At last, it is uncertain whether the findings in Tianjin city can be extrapolated to other districts.

\section{Conclusion}

The main finding in this study was that acupuncture treatment was efficacious and well tolerated in improving cognitive function and global clinical status. Acupuncture offers a promising and effective treatment option for $A D$ to physicians, with few adverse effects or contraindications.

\section{Abbreviations \\ AD: Alzheimer's disease; ADAS-ADL 23 : 23-Item Alzheimer's disease Cooperative Study Activities of Daily Living Scales; ADAS-cog: Alzheimer's disease Assessment Scale-Cognitive; ADL: Activity of Daily Living scale; AEs: Adverse events; AG: Acupuncture group; CIBIC-Plus: Clinician's Interview- Based Impression of Change-Plus; DG: Donepezil hydrochloride group; HAMD: 17-item Hamilton depression scale; HIS: Hanchinski ischemia scale; MMSE: Mini-mental State Examination; NPI: Neuropsychiatric Index; SAEs: Serious adverse events; T0: 4-week baseline; T1: 12-week treatment phase; T2: 12-week follow-up period; TCM: Traditional Chinese medicine; USA: united states of American; WHO: World Health Organization}

\begin{abstract}
Acknowledgments
We would like to express our sincere gratitude to research staff at the chronic disease prevention and control department of Simianzhong community hospital in Heping District, TianjinCity. We also wish to thank Yufei Cai, Gang Chen, Chunqing Feng, Qichao Hu, Xiaoxi Liu for their epidemiological investigation work, and Yu Fu, Lili Yin, Yanan Zhou, chuan Xiao, Bangqi Wu and Yuan Meng for their acupuncture treatment.
\end{abstract}

\section{Funding}

The study was supported by National Natural Science Foundation of China (81473766) and Key Project of Natural Science Foundation of Tianjin City (16JCZDJC36200).The funders had no role in study design, data collection and analysis, decision to publish, or preparation of the manuscript.

\section{Availability of data and materials}

All data generated or analyzed during this study are included in the manuscript. The datasets used and/or analyzed during the current study are available from the corresponding author upon reasonable request.

\section{Authors' contributions}

$J H, J Y$ and TY participated in the design of the study. TY, YJ, JS carried out the acupuncture manipulation, $X Z$ and $L Z$ evaluate scale, $K N$ analyzed the data, YJ and XZ wrote the manuscript. All authors read and approved the final manuscript.

\section{Ethics approval and consent to participate}

Ethics approval was granted by the Research Ethical Committee of First Teaching Hospital of Tianjin University of Traditional Chinese Medicine, China (Reference no.:TYLL2015[K]003) before the start of this study. A written informed consent was obtained from a legal representative of the participants prior to their involvement.

\section{Consent to publish}

Not applicable.

\section{Competing interests}

The authors declare that they have no competing interests.

\section{Publisher's Note}

Springer Nature remains neutral with regard to jurisdictional claims in published maps and institutional affiliations.

Received: 7 June 2017 Accepted: 15 December 2017

Published online: 29 December 2017

\section{References}

1. McKhann G, Drachman D, Folstein M, Katzman R, Price D, Stadlan EM. Clinical diagnosis of Alzheimer's disease: report of the NINCDS-ADRDA work group under the auspices of Department of Health and Human Services Task Force on Alzheimer's disease. Neurology. 1984;34(7):939-44.

2. Jindal H, Bhatt B, Sk S, Singh Malik J. Alzheimer disease immunotherapeutics: then and now. Human vaccines \& immunotherapeutics. 2014;10(9):2741-3.

3. Wang XP, Ding HL. Alzheimer's disease: epidemiology, genetics, and beyond. Neurosci Bull. 2008;24(2):105-9.

4. Reitz C, Mayeux R. Alzheimer disease: epidemiology, diagnostic criteria, risk factors and biomarkers. Biochem Pharmacol. 2014;88(4):640-51.

5. Qiu C, De Ronchi D, Fratiglioni L. The epidemiology of the dementias: an update. Current opinion in psychiatry. 2007;20(4):380-5.

6. Plassman BL, Langa KM, Fisher GG, Heeringa SG, Weir DR, Ofstedal MB, Burke JR, Hurd MD, Potter GG, Rodgers WL, et al. Prevalence of dementia in the United States: the aging, demographics, and memory study. Neuroepidemiology. 2007;29(1-2):125-32.

7. Zec RF, Burkett NR. Non-pharmacological and pharmacological treatment of the cognitive and behavioral symptoms of Alzheimer disease. NeuroRehabilitation. 2008;23(5):425-38.

8. Atri A, Shaughnessy LW, Locascio JJ, Growdon JH. Long-term course and effectiveness of combination therapy in Alzheimer disease. Alzheimer Dis Assoc Disord. 2008;22(3):209-21.

9. Lanctot KL, Herrmann N, Yau KK, Khan LR, Liu BA, Lou Lou MM, Einarson TR. Efficacy and safety of cholinesterase inhibitors in Alzheimer's disease: a meta-analysis. CMAJ: Canadian Medical Association journal = journal de l'Association medicale canadienne. 2003;169(6):557-64.

10. Shah S, Reichman WE. Treatment of Alzheimer's disease across the spectrum of severity. Clin Interv Aging. 2006;1(2):131-42.

11. Farlow MR, Miller ML, Pejovic V. Treatment options in Alzheimer's disease: maximizing benefit, managing expectations. Dement Geriatr Cogn Disord. 2008;25(5):408-22

12. Lee MS, Shin BC, Ernst E. Acupuncture for Alzheimer's disease: a systematic review. Int J Clin Pract. 2009;63(6):874-9.

13. Zhou J, Peng W, Xu M, Li W, Liu Z. The effectiveness and safety of acupuncture for patients with Alzheimer disease: a systematic review and meta-analysis of randomized controlled trials. Medicine. 2015;94(22):e933. 
14. Porsteinsson AP, Grossberg GT, Mintzer J, Olin JT, Memantine MEMMDSG. Memantine treatment in patients with mild to moderate Alzheimer's disease already receiving a cholinesterase inhibitor: a randomized, doubleblind, placebo-controlled trial. Curr Alzheimer Res. 2008;5(1):83-9.

15. Bassil N, Grossberg GT. Novel regimens and delivery systems in the pharmacological treatment of Alzheimer's disease. CNS drugs. 2009;23(4): 293-307.

16. Maher-Edwards G, Dixon R, Hunter J, Gold M, Hopton G, Jacobs G, Hunter J, Williams P. SB-742457 and donepezil in Alzheimer disease: a randomized, placebo-controlled study. International journal of geriatric psychiatry. 2011; 26(5):536-44.

17. Galasko D, Bennett D, Sano M, Ernesto C, Thomas R, Grundman M, Ferris S. An inventory to assess activities of daily living for clinical trials in Alzheimer's disease. The Alzheimer's disease cooperative study. Alzheimer Dis Assoc Disord. 1997;11(Suppl 2):S33-9.

18. Cummings JL, Mega M, Gray K, Rosenberg-Thompson S, Carusi DA, Gornbein J. The neuropsychiatric inventory: comprehensive assessment of psychopathology in dementia. Neurology. 1994;44(12):2308-14.

19. Gu W, Jin X, Zhang Y. Clinical observation on treatment of Alzheimer's disease by acupuncture. Chinese Acupuncture\&Moxibustion. 2014;34(12): 1156-60.

20. Wang Z, Liang P, Zhao Z, Han Y, Song H, Xu J, Lu J, Li K. Acupuncture modulates resting state hippocampal functional connectivity in Alzheimer disease. PLoS One. 2014:9(3):e91160

21. Wang Z, Nie B, Li D, Zhao Z, Han Y, Song H, Xu J, Shan B, Lu J, Li K. Effect of acupuncture in mild cognitive impairment and Alzheimer disease: a functional MRI study. PLoS One. 2012;7(8):e42730.

22. Liang P, Wang Z, Qian T, Li K. Acupuncture stimulation of Taichong (Liv3) and Hegu (L/4) modulates the default mode network activity in Alzheimer's disease. American journal of Alzheimer's disease and other dementias. 2014; 29(8):739-48.

23. Sutalangka C, Wattanathorn J, Muchimapura S, Thukham-Mee W, Wannanon $\mathrm{P}$, Tong-un T. Laser acupuncture improves memory impairment in an animal model of Alzheimer's disease. Journal of acupuncture and meridian studies. 2013:6(5):247-51.

24. Lin R, Chen J, Li X, Mao J, Wu Y, Zhuo P, Zhang Y, Liu W, Huang J, Tao J, et al. Electroacupuncture at the Baihui acupoint alleviates cognitive impairment and exerts neuroprotective effects by modulating the expression and processing of brain-derived neurotrophic factor in APP/PS1 transgenic mice. Mol Med Rep. 2016;13(2):1611-7.

25. Cheng H, Yu J, Jiang Z, Zhang X, Liu C, Peng Y, Chen F, Qu Y, Jia Y, Tian Q et al. Acupuncture improves cognitive deficits and regulates the brain cell proliferation of SAMP8 mice. Neurosci Lett. 2008;432(2):111-6.

26. Zhao L, Jia Y, Yan D, Zhou C, Han J, Yu J. Aging-related changes of triose phosphate isomerase in hippocampus of senescence accelerated mouse and the intervention of acupuncture. Neurosci Lett. 2013;542:59-64.

27. Nie K, Zhang XZ, Zhao L, Jia YJ, Han JX. Effect of acupuncture on transmembrane signal pathway in AD mice: an analysis based on lipid-raft proteomics. Zhongguo Zhong xi yi jie he za zhi Zhongguo Zhongxiyi jiehe zazhi $=$ Chinese journal of integrated traditional and Western medicine. 2014;34(8):991-6

28. J S. The organization and function of endogenous antinociceptive systems. Prog Neurobiol.1996;50(1):49-81

29. Irnich D BA. Neurobiological mechanisms of acupuncture analgesia. Schmerz. 2002,16(2):93-102

30. Macpherson H. Pragmatic clinical trials. Complementary therapies in medicine. 2004:12(2-3):136-40.

31. Frölich $L, K T$, Berger FM. Treatment with donepezil in Alzheimer patients with and without cerebrovascular disease. J Neurol Sci. 2002 Nov 15; 2002(203-204):137-9.

32. Doody RS, Geldmacher DS, Gordon B, Perdomo CA, Pratt RD, Donepezil Study G. Open-label, multicenter, phase 3 extension study of the safety and efficacy of donepezil in patients with Alzheimer disease. Arch Neurol. 2001; 58(3):427-33.

\section{Submit your next manuscript to BioMed Central and we will help you at every step:}

- We accept pre-submission inquiries

- Our selector tool helps you to find the most relevant journal

- We provide round the clock customer support

- Convenient online submission

- Thorough peer review

- Inclusion in PubMed and all major indexing services

- Maximum visibility for your research

Submit your manuscript at www.biomedcentral.com/submit

) Biomed Central 